\title{
Quantification of the dynamics of antibody response to malaria to inform sero-surveillance in pregnant women
}

A. D. V. Tharkeshi T. Dharmaratne', Saber Dini ${ }^{1}$, Katherine O'Flaherty ${ }^{2}$, David J. Price ${ }^{1,3}$, James Beeson ${ }^{2,4,5}$, Rose McGready ${ }^{6,7}$, Francois Nosten ${ }^{6,7}$, Freya J. I. Fowkes ${ }^{1,2,8}$, Julie A. Simpson ${ }^{1 *+}$ (D) and Sophie G. Zaloumis ${ }^{1+}$

\begin{abstract}
Background: Malaria remains a major public health threat and tools sensitive to detect infections in low malaria transmission areas are needed to progress elimination efforts. Pregnant women are particularly vulnerable to malaria infections. Throughout pregnancy they access routine antenatal care, presenting a unique sentinel population to apply novel sero-surveillance tools to measure malaria transmission. The aim of this study was to quantify the dynamic antibody responses to multiple antigens during pregnancy so as to identify a single or multiple antibody response of exposure to malaria in pregnancy.
\end{abstract}

Methods: This study involved a secondary analysis of antibody responses to six parasite antigens [five commonly studied merozoite antigens and the variant surface antigen 2-chondroitin sulphate A (VAR2CSA), a pregnancy-specific erythrocytic antigen] measured by enzyme-linked immunosorbent assay (ELISA) over the gestation period until delivery (median of 7 measurements/woman) in 250 pregnant women who attended antenatal clinics located at the ThaiMyanmar border. A multivariate mixture linear mixed model was used to cluster the pregnant women into groups that have similar longitudinal antibody responses to all six antigens over the gestational period using a Bayesian approach. The variable-specific entropy was calculated to identify the antibody responses that have the highest influence on the classification of the women into clusters, and subsequent agreement with grouping of women based on exposure to malaria during pregnancy.

Results: Of the 250 pregnant women, 135 had a Plasmodium infection detected by light microscopy during pregnancy (39\% Plasmodium falciparum only, 33\% Plasmodium vivax only and 28\% mixed/other species), defined as cases. The antibody responses to all six antigens accurately identified the women who did not have a malaria infection detected during pregnancy (93\%, 107/115 controls). Antibody responses to P. falciparum merozoite surface protein 3 (PFMSP3) and P. vivax apical membrane antigen 1 (PVAMA1) were the least dynamic. Antibody responses to the antigens $P$. falciparum apical membrane antigen 1 (PFAMA1) and PFVAR2CSA were able to identify the majority of the cases more accurately $(63 \%, 85 / 135)$.

Conclusion: These findings suggest that the combination of antibodies, PFAMA1 and PFVAR2CSA, may be useful for sero-surveillance of malaria infections in pregnant women, particularly in low malaria transmission settings. Further

\footnotetext{
*Correspondence: julieas@unimelb.edu.au

†Julie A. Simpson and Sophie G. Zaloumis have equal contribution

${ }^{1}$ Centre for Epidemiology and Biostatistics, Melbourne School

of Population and Global Health, The University of Melbourne,

Melbourne, Australia

Full list of author information is available at the end of the article
}

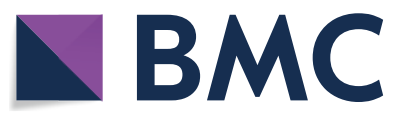

(c) The Author(s) 2022. Open Access This article is licensed under a Creative Commons Attribution 4.0 International License, which permits use, sharing, adaptation, distribution and reproduction in any medium or format, as long as you give appropriate credit to the original author(s) and the source, provide a link to the Creative Commons licence, and indicate if changes were made. The images or other third party material in this article are included in the article's Creative Commons licence, unless indicated otherwise in a credit line to the material. If material is not included in the article's Creative Commons licence and your intended use is not permitted by statutory regulation or exceeds the permitted use, you will need to obtain permission directly from the copyright holder. To view a copy of this licence, visit http://creativecommons.org/licenses/by/4.0/. The Creative Commons Public Domain Dedication waiver (http://creativeco mmons.org/publicdomain/zero/1.0/) applies to the data made available in this article, unless otherwise stated in a credit line to the data. 
investigation of other antibody markers is warranted considering these antibodies combined only detected $63 \%$ of the malaria infections during pregnancy.

Keywords: Malaria, Pregnancy, Antibodies, PfAMA1, PfEBA175, PfMSP2, PfMSP3, PVAMA1, PfVAR2CSA, Longitudinal data

\section{Background}

Malaria is a major infectious disease causing around 229 million clinical cases and 409,000 deaths globally in 2019 [1]. Pregnant women are particularly vulnerable to malaria infection, as well as presenting with more severe symptomatic infections [2]. Each year, around 125 million pregnant women, living in malaria endemic countries, are at risk of malaria infection [3, 4]. Malaria in pregnancy poses substantial risks to the pregnant woman and their baby, increasing the risk of maternal anaemia, hypertensive disorders, miscarriage, stillbirth and neonatal death and as such there are several prevention and treatment strategies provided to women attending antenatal care to reduce the burden of malaria in pregnancy $[5,6]$. Pregnant women routinely attending antenatal care are also considered an easy-access population which can serve as sentinel surveillance populations to estimate malaria transmission [7].

The development of novel serological surveillance (sero-surveillance) tools for use in sentinel populations of pregnant women is a potential powerful technique for detecting recent and ongoing malaria infections, and monitoring malaria transmission [8, 9]. This is particularly pertinent in low malaria transmission settings such as Southeast Asia, where parasite density is often low and standard surveillance methods (microscopy and rapid diagnostic tests) have reduced sensitivity with low density, submicroscopic and asymptomatic infections [10]. Sero-surveillance tools have the potential to increase the time window for detecting an infection, and thereby increasing the resolution of surveillance [9]. Antibodies targeting blood-stage antigens, predominantly relatively conserved antigens expressed on the merozoites, have been the focus of sero-surveillance studies in nonpregnant populations $[9,11]$. In pregnant women, serological studies have also investigated antibody responses to the pregnancy-specific Plasmodium falciparum antigen (PfVAR2CSA), which is expressed on the surface of infected erythrocytes (IEs), and mediates sequestration of $P$. falciparum in the placenta via binding to placental chondroitin sulphate A (CSA) receptors [12, 13]. Antibodies specific for PfVAR2CSA may reduce the accumulation of the IEs in the placenta [14]. High antibody levels against PfVAR2CSA can be acquired with successive pregnancies [15], potentially reducing the susceptibility to falciparum malaria in multigravida women by preventing or clearing parasite sequestration in the placenta [16]. While numerous studies have investigated PfVAR2CSA antibodies in pregnant women as markers of infection [17], few studies have incorporated non-pregnancy specific antibodies $[18,19]$, such as those targeting merozoite antigens, and none have considered the combined effects of these antibodies.

The aim of this study was to quantify the dynamic antibody responses to multiple blood-stage antigens (merozoite and PfVAR2CSA) during pregnancy to determine which, if any, of the antibody response(s) are biomarker(s) of exposure to malaria in pregnancy that could subsequently be used for sero-surveillance. Longitudinal antibody responses to both $P$. falciparum and Plasmodium vivax previously measured by ELISA in pregnant women attending antenatal clinics on the ThaiMyanmar border [18], a low malaria transmission setting $[20,21]$, were jointly analysed to account for the correlations between the antibodies to inform sero-surveillance approaches in pregnant women.

\section{Methods \\ Study population and design}

The study population was pregnant women attending antenatal clinics (ANCs) at the Shoklo Malaria Research Unit (SMRU) [22, 23], where malaria transmission is low and peaks between May and September. The ANCs were located in the Maela refugee camps, where approximately $90 \%$ of women attend on a weekly basis [23].

Details of the nested case-control study design and procedures have been published previously [18]. In brief, participants were identified from 1000 Karen women who were enrolled in a placebo randomized controlled trial of chloroquine prophylaxis against $P$. vivax infection during pregnancy from November 1998 through January 2000 [24]. Samples were obtained weekly from the women for Plasmodium species infection detection by microscopic examination of blood smears and fortnightly for serum sample collection. Case subjects were women with Plasmodium infection detected by light microscopy at any time during pregnancy during the trial $(n=136)$. Of the 864 women with no detectable parasitaemia at any time while pregnant during the trial, 331 were randomly selected to be control subjects (3:1 ratio). All available serum samples from the 136 case subjects were included for quantification of antibodies to multiple antigens over 
time [18]. A subset of 115 control subjects was selected for longitudinal antibody determination based on IgG responses to $P$. falciparum (3D7) schizont extract measured by ELISA at enrollment. The 115 controls were selected as follows. All available control enrollment samples (320 of the 331 randomly selected controls had serum samples measured at enrollment) were first tested for total IgG in response to P. falciparum schizont protein extract. A cut-off threshold for seropositivity to schizont extract was set to the mean +3 standard deviations of the IgG responses to schizont extract for 8 negative controls (non-exposed Melbourne donors). The subset of 115 controls consisted of 78 individuals seropositive to schizont extract at enrollment (total of $\mathrm{n}=572$ samples included) together with 37 randomly selected individuals who were seronegative to schizont extract at enrollment (total of $\mathrm{n}=323$ samples included) (Fig. 1). See Additional file 1, Fowkes et al. [18] for further details. Of note, the nested case-control study design for the selected serum samples was not designed for this analysis.

\section{Antibody determination}

High throughput enzyme-linked immunosorbent assay (ELISA) $[18,25]$ was used to determine the total IgG magnitude (measured as optical density (OD) values) of $P$. falciparum merozoite antigens and one $P$. vivax merozoite antigen (apical membrane antigen-1 [PvAMA1] ectodomain based on the SalI sequence, PVX_ 092275) [26]. P. falciparum antigens included were (from 3D7 reference sequence): apical membrane antigen 1 (PfAMA1; ectodomain) [27], erythrocyte binding antigen 175 (PfEBA175; region 3-5) [28], merozoite surface protein 2 (PfMSP2, full length) [27], merozoite surface protein 3 (PfMSP3; amino acids 154-249) [29, 30], schizont extract, PfVAR2CSA (DBL5 $\varepsilon$ domain), (Additional file 1: Supplementary methods and Fowkes et al. [18]). Schizont lysate was prepared from $P$. falciparum (3D7) cultures according to previously described methods [27].

\section{Statistical analysis}

Patient characteristics at baseline were summarized using median (25th-75th percentiles) for continuous variables or frequency (\%) for categorical variables.

A multivariate mixture linear mixed model was used to identify clusters (i.e., latent classes) of pregnant women that have similar antibody responses to all six antigens over gestational age [31]. To construct a multivariate mixture linear mixed effects model, first a linear mixed effects model was specified for each of the six antibody responses. The following covariates were included as fixed effects for each antibody response: age (years), primigravidae ( 1 if primigravidae and 0 if multigravida), treatment arm (1 if given chloroquine (CQ) as prophylaxis at enrolment and 0 if given a placebo) and having a history of malaria prior to enrolment ( 1 if exposed to malaria at least once prior to enrolment and 0 otherwise). To capture the between-subject variability in the six antibody responses over gestational age, a random intercept and random slope for gestational age were included in the linear mixed effects model for each antibody response. The random intercepts and slopes for each response and woman (i.e., 12 random effects per woman) are assumed to follow a mixture of multivariate normal distributions.

The mixAK package in $\mathrm{R}$ [32] was used to fit the multivariate mixture linear mixed effects model to the six antibody response profiles available from each of the 250 women (135 cases and 115 controls) in this study. The mixAK package adopts a Bayesian approach to inference and implements a block Gibbs sampler with Metropolis-Hastings steps to sample parameter values from the posterior distribution (Additional file 1, Komárek and Komárková (2013, Appendix B) [31]). Weakly informative prior hyperparameters in the mixAK package were used (Additional file 1, Komárek and Komárková (2013, Appendix A) [31]). Two chains were initialized. The first 500 parameter values sampled for each chain were discarded as burn-in and an additional 5,000,000 parameter values (in total for both chains) were sampled after burn-in. Every 50th iteration after burn-in was kept, resulting in 100,000 (50,000 per chain) samples per parameter for calculation of posterior summaries. Results are presented as the posterior median (50th percentile) and 95\% credible interval, calculated as the 2.5th and 97.5th percentiles of the 100,000 samples for each parameter. Traceplots were examined to assess whether the 50,000 parameter draws from each chain had appropriately converged.

The number of clusters was selected by fitting a mixture model assuming each of 1-4 clusters. The number of clusters was selected according to the model that produced the lowest penalized expected deviance and/or greatest shift of the posterior distribution of deviances to lower values [31]; Additional file 1: Supplementary methods, Statistical Analyses.

The posterior probability of a woman belonging to a cluster (posterior class probability) was calculated at each iteration of the fitting algorithm and these probabilities were used to assign a woman to a cluster as follows. First, a woman was assigned to the cluster which had the highest median posterior class probability. Second, the woman remained in the cluster if the lower limit of the 95\% credible interval for the posterior class probability exceeded 0.5 ; otherwise, the woman was considered unclassified (Additional file 1: Supplementary methods, Statistical Analyses). 


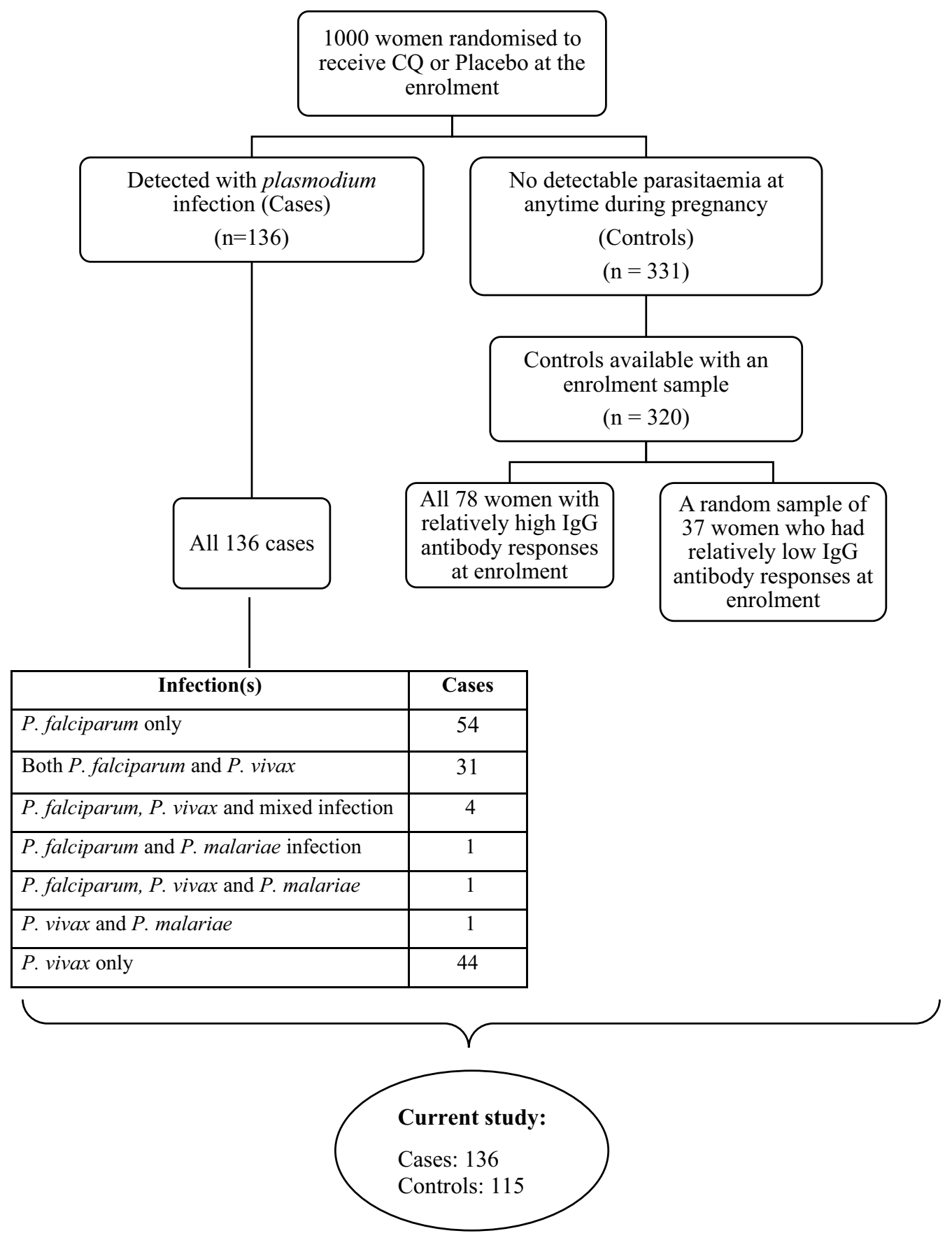

Fig. 1 Flow diagram of cohort selection for the current study

The variable-specific entropy was calculated to identify the antibody responses that have the highest influence on the classification of the women into clusters. The variable-specific entropy indicates how well the antibody response to a single antigen predicts the classification based on antibody responses to all six antigens, and ranges from 0 to 1 [33]. Antibody responses with variable-specific entropy values close to 1 drive the classification of women into clusters (Additional file 1: Supplementary methods, Statistical Analyses).

To compare and identify the best antibodies for classifying women as being a case (defined as malaria detected by microscopy during pregnancy) or control, additional univariate and pairwise multivariate mixture linear 
mixed-effects modelling were performed with the number of clusters set at two groups, and the proportion of cases and controls classified in the high and low antibody response groupings calculated.

\section{Results}

\section{Patient characteristics}

A total of 1692 samples were included in the analysis; 727 samples were available from the 135 cases (1 case excluded because only an enrolment sample was available) and 965 from the 115 controls. For the 135 cases, $39 \%$ had $P$. falciparum infections only, 33\% P. vivax only and $28 \%$ mixed/other species, see Additional file 1: Table S1 for number and species of infection(s) recorded during pregnancy.

Table 1 presents the baseline characteristics of the 135 cases (women with $P$. falciparum and/or P. vivax detected by microscopy during pregnancy) and 115 controls (women without detected Plasmodium infection). The proportion of cases in their first pregnancy $(22.2 \%)$ at enrolment was around twice of that in the control group (13.9\%). Anaemia was more prevalent among cases at enrolment (23.7\%), compared to controls (9.6\%). The proportion of women who had received chloroquine prophylaxis (only to prevent vivax episodes) [24] was slightly higher for the control group (47.8\% vs $41.5 \%)$. For both cases and controls, most women were enrolled in the trial during their first trimester (cases $75.6 \%$ and controls $85.2 \%)$. Nearly all controls were residing in refugee camps (99.1\%), while less than half of the cases $(45.2 \%)$ were living in refugee camps during pregnancy (the remaining cases were residing in villages south of the Mae Sot township).

More than half of the cases (55.6\%) had a documented history of malaria at the SMRU ANCs (any Plasmodium spp.; $37.9 \%$ P. falciparum and $24.2 \%$ P. vivax) prior to enrollment compared with $36.5 \%$ of the controls $(27.8 \%$ P. falciparum and $11.3 \%$ P. vivax).

\section{Antibody dynamics over gestation}

For all six antigens, the antibody response profiles of pregnant women who were infected with malaria during the trial (cases) tended to be maintained at higher levels compared to women who were free from malaria (controls) (Fig. 2). However, substantial variation in the antibody profiles of PfAMA1, PfEBA175, PfMSP2 and PfVAR2CSA was observed, irrespective of exposure to malaria (Fig. 2A-C, F). For PfMSP3 and PvAMA1, the antibody profiles tended to remain low over gestation for most controls, while the antibody responses for the

Table 1 Distribution of patient characteristics at enrollment for cases and controls

\begin{tabular}{|c|c|c|}
\hline Characteristic & $\begin{array}{l}\text { Cases } \\
(n=135)\end{array}$ & $\begin{array}{l}\text { Controls } \\
(n=115)\end{array}$ \\
\hline Age (years), median (IQR) & $24(20-30)$ & $26(22-32)$ \\
\hline Gravidity, median (IQR) & $3(2.0-4.5)$ & $3(2.0-5.0)$ \\
\hline Primigravida, n (\%) & $30(22.2)$ & $16(13.9)$ \\
\hline Multigravida, n (\%) & $105(77.8)$ & $99(86.1)$ \\
\hline Parity, median (IQR) & $1(0-3)$ & $2(1-4)$ \\
\hline Haematocrit (\%), median (IQR) & $32.5(30-35)$ & $34.0(32-36)$ \\
\hline Anaemia ${ }^{a}, \mathrm{n}(\%)$ & $32(23.7)$ & $11(9.6)$ \\
\hline Residence in refugee camp & $61(45.2)$ & $114(99.1)$ \\
\hline Received chloroquine prophylaxis, n (\%) & $56(41.5)$ & $55(47.8)$ \\
\hline Estimated Gestational Age ${ }^{\mathrm{b}}$ (weeks), median (IQR) & $9.7(7-14)$ & $9.4(7.6-11.6)$ \\
\hline \multicolumn{3}{|l|}{ Trimester } \\
\hline 1 (<14 weeks), n (\%) & $102(75.6)$ & $98(85.2)$ \\
\hline 2 (14 to < 28 weeks), n (\%) & $31(23)$ & $17(14.8)$ \\
\hline 3 (28 week or more), n (\%) & $2(1.5)$ & $0(0.0)$ \\
\hline Plasmodium spp. before enrolment ${ }^{c}, \mathrm{n}(\%)$ & $75(55.6)$ & $42(36.5)$ \\
\hline Infected with P. falciparum ${ }^{\mathrm{d}}, \mathrm{n}(\%)$ & $50(37.9)$ & $32(27.8)$ \\
\hline Infected with P. vivax ${ }^{\mathrm{d}}, \mathrm{n}(\%)$ & $32(24.2)$ & $13(11.3)$ \\
\hline Follow up (weeks), median (range) & $28.9(22.6-32.1)$ & $30.9(28.3-32.4)$ \\
\hline
\end{tabular}

${ }^{a}$ Haematocrit $<30 \%$

${ }^{\mathrm{b}}$ Determined at enrolment

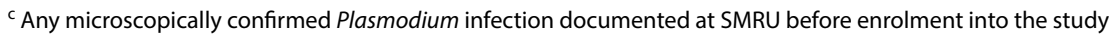

${ }^{d}$ Includes women that have a history of both P. falciparum and $P$. vivax infections. Hence, the summation of $P$. falciparum ${ }^{d}$ and $P$. vivax ${ }^{d}$ women does not add up to Plasmodium spp. before enrolment ${ }^{c}$ 

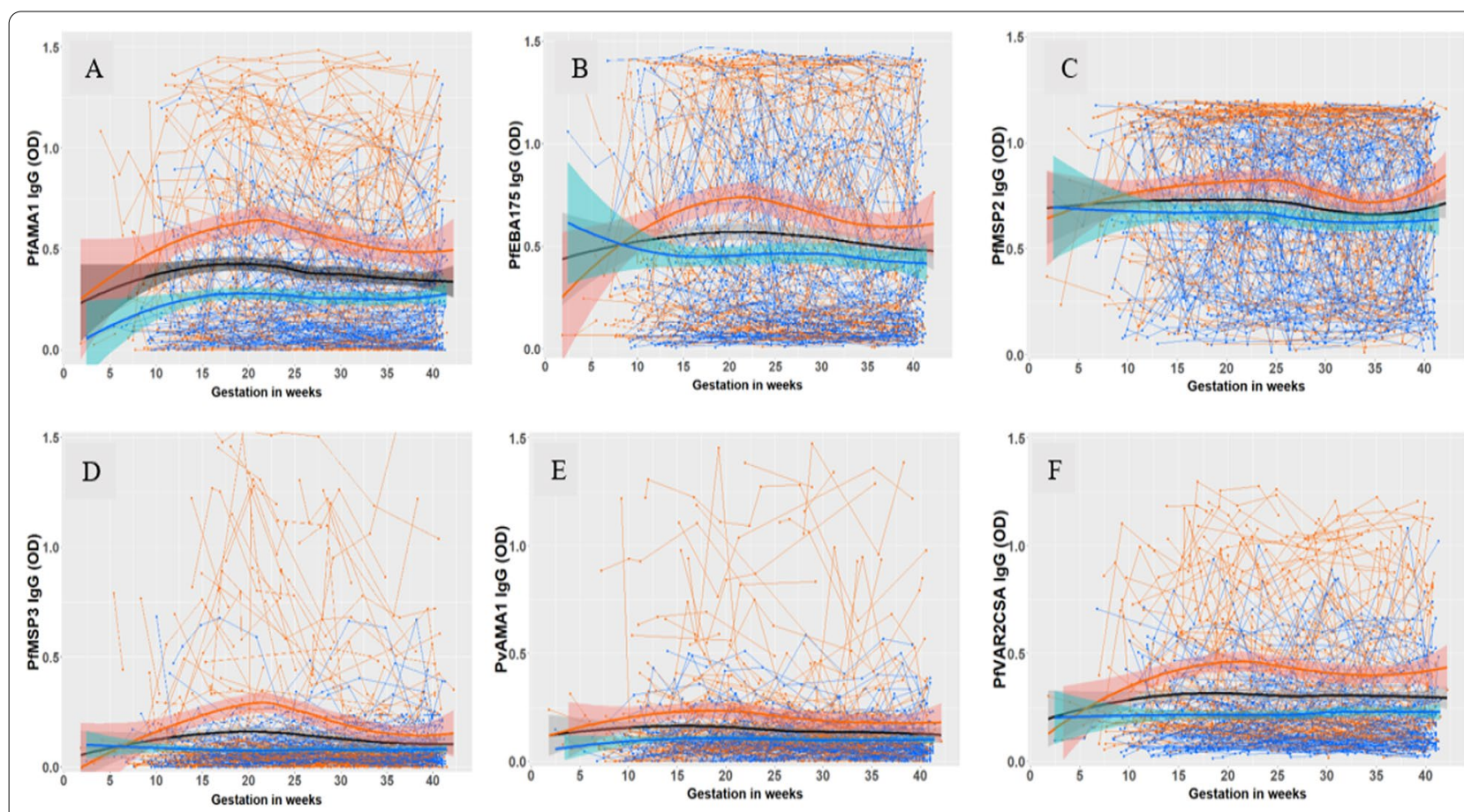

Fig. 2 Longitudinal antibody levels against six antigens for all women. Spaghetti plots $\mathbf{A}-\mathbf{F}$ represent the antibody profiles of PfAMA1, PfEBA175, PFMSP2, PFMSP3, PVAMA1 and PFVAR2CSA, respectively. The antibody levels of the pregnant women exposed to malaria (cases) and free from malaria by microscopy (controls) are represented by orange and blue, respectively. Locally estimated scatterplot smoothing (LOESS) curves for all pregnant women (in black) and for each exposure group are superimposed on each spaghetti plot. The shaded area around each LOESS curve represents the 95\% confidence interval (Cl). Of note, the $\mathrm{Y}$ axes of the plots of $\mathbf{A}, \mathbf{B}$ and $\mathbf{D}$ are truncated at 0; the Cls did extend to negative values due to limited information in the early period of gestation. OD optical density

cases exhibited greater variability (Fig. 2D-E). The controls consisted of 78 individuals' seropositive to schizont extract at enrolment together with 37 randomly selected individuals who were seronegative to schizont extract at enrolment. Additional file 1: Fig. S1 shows that the longitudinal antibody responses of these two control subgroups are similar, with slightly higher values in PfAMA1 and PfMSP2 for those seropositive to schizont extract.

The behaviour of the longitudinal antibody profiles by gravidity (primigravidae versus multigravidae) of the pregnant women are presented in Additional file 1: Fig. S2, and suggest similar between- and within-individual variation in levels for primigravid and multigravida women.

\section{Classification of pregnant women}

The best fitting multivariate mixture linear mixed effects model according to the penalized expected deviance and the posterior distribution of the deviances classified the six antibody profiles from each of the 250 pregnant women into two clusters (Additional file 1: Supplementary methods, Sects. 1-5): 186 into Cluster 1 and 55 into Cluster 2, with 9 unclassified (see Additional file 1:
Table S3 for baseline characteristics of pregnant women by cluster group). The antibody response profiles allocated to each cluster are shown in Fig. 3 and demonstrate that the clustering method has clearly differentiated Cluster 1 as a group of pregnant women with antibody profiles maintained at relatively low levels or dynamic low-medium levels across all 6 antibodies, from Cluster 2 as those who had relatively high or dynamic mediumhigh antibody levels over gestational age. As such, herein clusters 1 and 2 are referred to as "low immune" and "high immune" groups, respectively.

\section{Association between maternal factors and antibody responses}

Posterior summaries for the fixed effect and population average intercept and slope parameters of the multivariate mixture linear mixed effects model are presented in Table 2. For most antigens, the mean change in antibody responses increased with age (per year) and were higher for those pregnant women that received chloroquine prophylaxis; the exceptions were the mean change in antibody titer to PvAMA1 decreased by -0.001 with age (per year) $(95 \%$ Credible Interval 


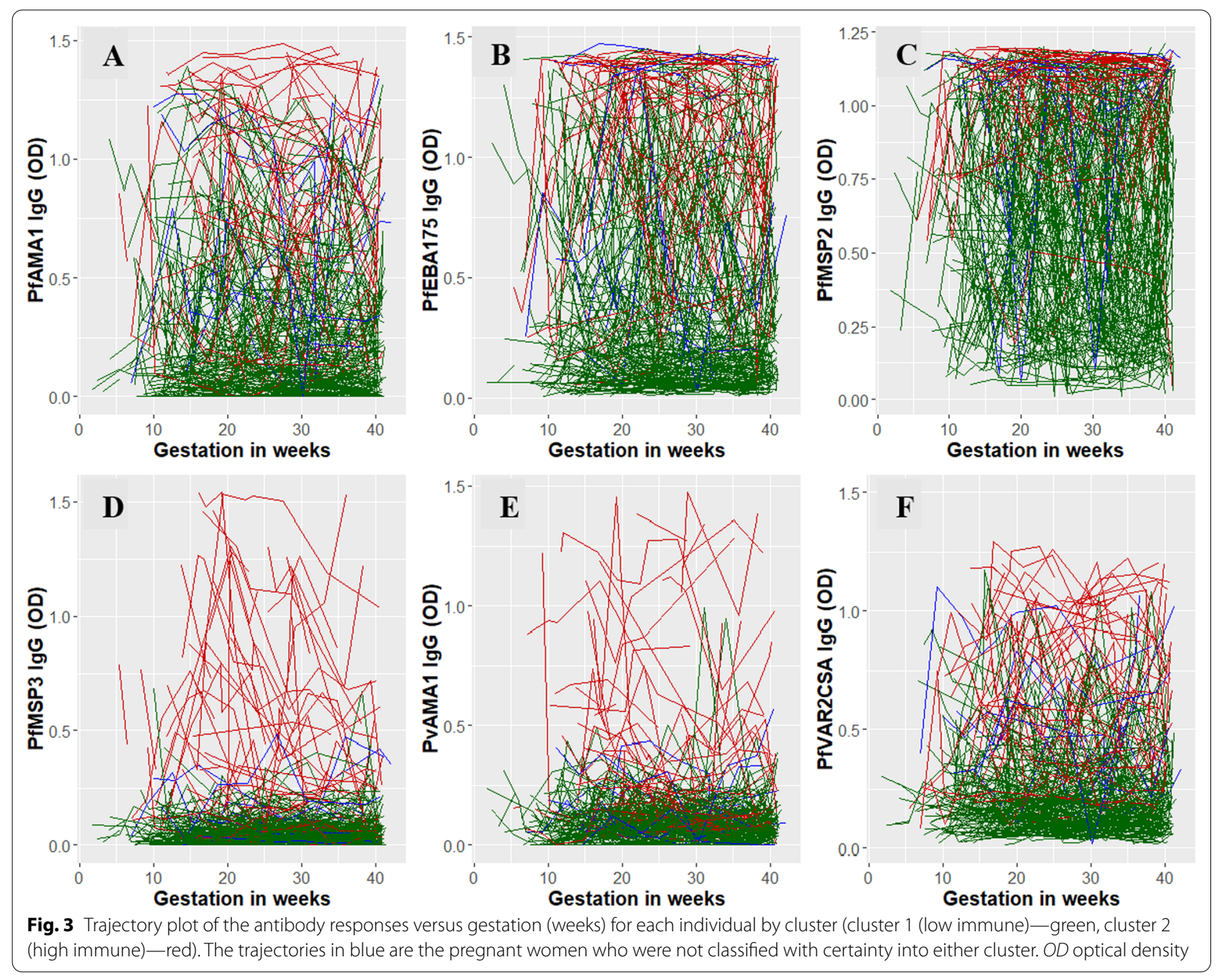

$(\mathrm{CrI}):-0.002,0.0004))$ and the mean antibody titer to PfMSP3 were -0.003 (95\% CrI: - 0.019, 0.013) lower for those that received chloroquine prophylaxis compared to those that did not. Mean antibody responses to PfAMA1, PfMSP2 and PfMSP3 were increased for primigravidae compared to multigravidae women. For pregnant women with a history of malaria, only mean antibody responses to the antigen PvAMA1 were increased (0.012 (95\% CrI: - 0.005, 0.028)) compared to women without a history of prior infection(s). Of note, the majority of the limits of the $95 \%$ CrIs ranged from decreasing to increasing changes in mean antibody responses so the associations with the maternal factors described above are weak.

The posterior medians for the population average slope indicate that, on average, antibody responses to PfAMA1 and PvAMA1 decreased with gestational age in both the low and high immune groups. For PfEBA175, PfMSP2 and PfVAR2CSA, the posterior medians for the population average slope indicated that, on average, antibody responses decreased with gestational age in the low immune group but increased with gestational age in the high immune group; these trends were reversed for antibody responses to PfMSP3.

\section{Influence of specific antibodies on the classification into high and low immunity groups}

The variable-specific entropy for the antibody responses to the six antigens are given in Table 3. Antibody responses to the antigen PfMSP2 played a lesser role in cluster allocation (lowest entropy $=0.763$ ), whereas the relatively high entropy values for PfMSP3 (0.949) and PvAMA1 (0.935) indicate that the allocation of women to the low or high immune group was predominantly 


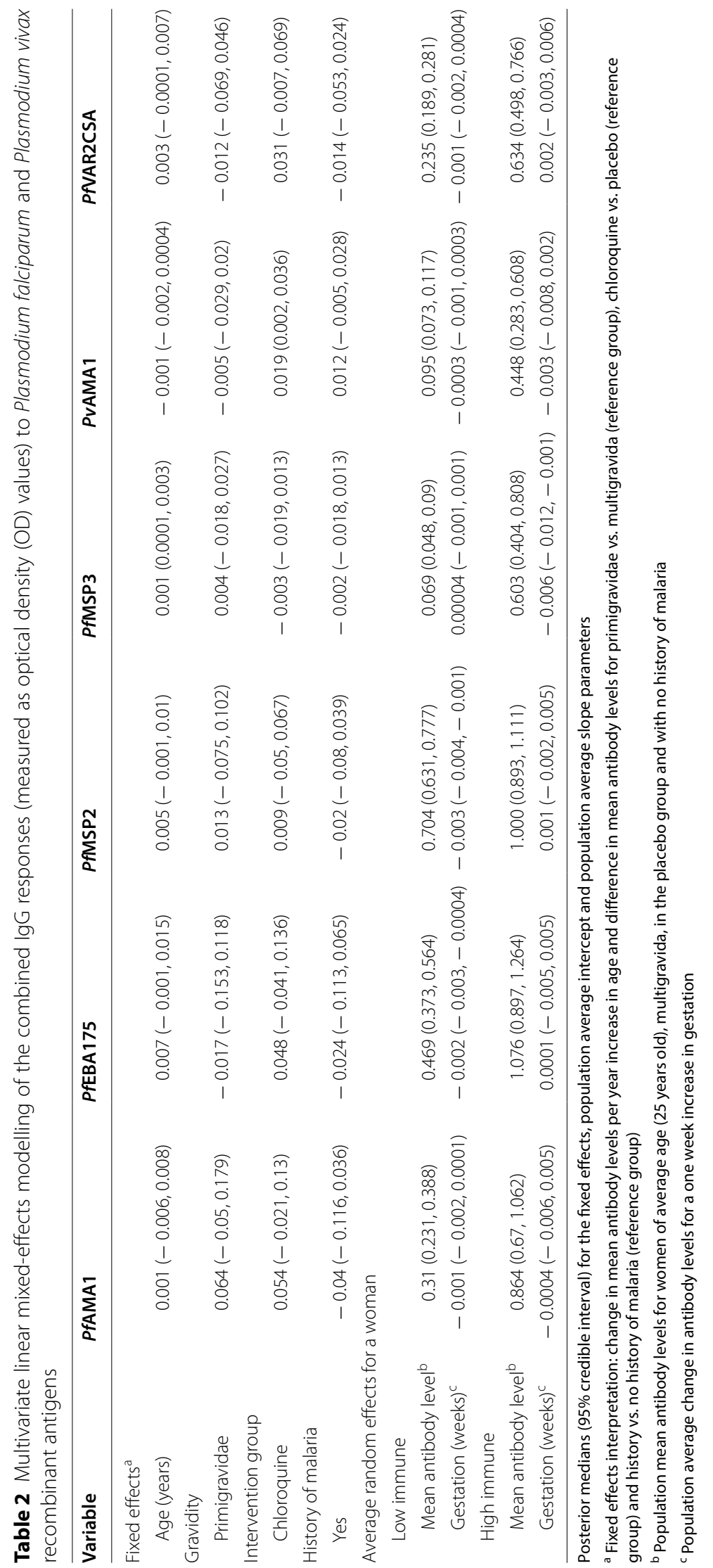


Table 3 Influence of specific antibodies on the classification into high and low immunity profiles

\begin{tabular}{ll}
\hline Antibody & Entropy value \\
\hline PFMSP3 & 0.949 \\
PVAMA1 & 0.935 \\
PFVAR2CSA & 0.873 \\
PFAMA1 & 0.844 \\
PFEBA175 & 0.817 \\
PFMSP2 & 0.763 \\
\hline
\end{tabular}

${ }^{a}$ The entropy is a value between 0 and 1 and values closer to 1 indicate that an antibody highly contributes towards the classification of women to cluster 1 or 2. The influence on classification declines as the entropy value declines

determined by their antibody responses to these antigens. Therefore, additional analyses were performed to assess the importance of including both PfMSP3 and PvAMA1 antibodies in allocating a woman to the low or high immune group. These analyses found that at least one of the two antigens, PvAMA1 or PfMSP3, is necessary for correctly grouping pregnant women with low antibody profiles (Additional file 1: Supplementary methods, Sect. 7).

\section{Antibodies that best identify malaria cases}

Using all six antibody responses, the multivariate model performed well for the controls by classifying them into the low immune group (107 of 111 controls, 96.4\%); whereas the cases were poorly identified by the antibody responses to the six antigens; 51 (39\%) and 79 (61\%) cases classified in high and low immune groups, respectively $(9$ unclassified -4 controls and 5 cases).
Analyses including each antigen separately (i.e. univariate linear mixed-effects modelling), found antibody responses to the antigen PfAMA1 were best able to discriminate between cases and controls and classified $66 \%$ of controls in the low immune group (77 out of 115 controls) and $55 \%$ of cases in the high immune group (74 out of 135 cases) (Table 4). The model fit to antibody responses to PvAMA1 was best at identifying the controls (95\%, 109 of 115 controls), followed by PfMSP3 (90\%, 103 of 115 controls). The model fit to antibody responses to PfEBA175 was best at identifying the majority of the cases $(60 \%, 81$ of 135 cases), followed by PfAMA1 (55\%, 74 of 135 cases). Although the model fits to antibody responses to PvAMA1 and PfEBA175 were best at identifying controls and cases, respectively, they were unable to accurately classify the pregnant women in the other exposure group (Table 4). Although antibodies are acquired to VAR2CSA from exposure to malaria infection in pregnancy, antibodies to VAR2CSA did not perform as well as several merozoite antigens.

Pairwise analyses of the antibody responses were further explored considering the top 3 antibodies which correctly identified $>55 \%$ of all women (cases and controls) into the expected cluster in the univariate analyses, i.e. PfAMA1, PfVAR2CSA and PfEBA175 (Table 4). The results of selected pairwise multivariate analyses (Additional file 1: Tables S6, S7) indicate that antibody responses to both PfAMA1 and PfVAR2CSA were best at identifying the cases as most women were classified into the high immune group. This combination classified $63 \%$ (73 of 115) of the controls into the low immune group and $63 \%$ (85 of 135) of the cases into the high immune group. For classification of falciparum and

Table 4 Performance of classifying controls to Cluster $1^{a}$ and cases to Cluster $2^{b}$ based on univariate analyses ${ }^{c}$

\begin{tabular}{|c|c|c|c|}
\hline & $\begin{array}{l}\text { Number of controls }{ }^{d} \text { classified into } \\
\text { Cluster } 1(\%)\end{array}$ & $\begin{array}{l}\text { Number of } \text { cases }^{\mathrm{e}} \text { classified into Cluster } \\
2(\%)\end{array}$ & $\begin{array}{l}\text { Total women classified into } \\
\text { the expected Cluster }{ }^{f}(\%)\end{array}$ \\
\hline PfAMA1 & 76 (66.1) & $74(54.8)$ & $150(60)$ \\
\hline PfVAR2CSA & $86(74.8)$ & $57(42.2)$ & $143(57.2)$ \\
\hline PfEBA175 & $61(53.0)$ & $81(60.0)$ & $142(56.8)$ \\
\hline PVAMA1 & $109(94.8)$ & $28(20.7)$ & $137(54.8)$ \\
\hline PFMSP3 & 103 (89.6) & $33(24.4)$ & $136(54.4)$ \\
\hline PFMSP2 & $63(54.8)$ & $67(49.6)$ & $130(52)$ \\
\hline
\end{tabular}

${ }^{a}$ Cluster 1 (low immunity group)

${ }^{\mathrm{b}}$ Cluster 2 (high immunity group)

${ }^{c}$ Antibodies are ordered from the highest to the lowest percentage of classifying pregnant women into the expected cluster

d Out of 115 controls

e Out of 135 cases

fComputed based on the decision made that Cluster 1 would represent controls and Cluster 2 would represent cases. The sum of controls classified into Cluster 1 and cases classified into Cluster 2 were then divided by the total pregnant women, i.e. 250 to obtain the percentage 
vivax infections, antibody responses to PfAMA1 and PfVAR2CSA classified the majority of $P$. falciparum only (81\% [43/53]) and both P. falciparum and P. vivax (77\% [24/31]) infections as cases, but performed poorly at classifying $P$. vivax only infections as cases $(27 \%[12 / 44])$ (Additional file 1: Table S8).

\section{Discussion}

Pregnant women may serve as an accessible sentinel population to estimate the burden of malaria [34] and potentially for sero-surveillance studies to detect infections. In this cohort, antibody responses to the malaria parasite were highly dynamic, varying greatly within and between women during pregnancy. Modelling the longitudinal antibody response to six different antigens simultaneously found that pregnant women were classified into two major clusters, high immune and low immune response groupings, where $96 \%$ of the women who did not have a malaria infection detected during pregnancy had a low immune response. Combined antibody responses to the antigens PfAMA1 and PfVAR2CSA were best for identifying exposure to malaria during pregnancy, suggesting that these two antibodies may be good candidates for sero-surveillance of malaria infections in pregnant women.

Antibodies specific for Plasmodium spp. blood-stage antigens, in particular PfVAR2CSA, are an attractive candidate for sero-surveillance of malaria in pregnancy as they have been demonstrated as a biomarker of recent exposure to malaria during pregnancy (systematically reviewed in [17]) and to monitor changes in malaria transmission over time [35]. However, the antibody to PfVAR2CSA when used on its own was not the best marker of malaria infection in pregnancy. A combination of antibodies to PfVAR2CSA and the merozoite antigen PfAMA1 was the most accurate indicator of exposure to malaria during pregnancy. Our results show that the overall immunity corresponding to the $P$. falciparum parasite is positively correlated with increasing age, potentially due to increased lifetime exposure to malaria [36, 37]. No strong correlation with gravidity was observed, even with the pregnancy-specific immunity (PfVAR2CSA), which may reflect the specific epidemiology of malaria in pregnancy in low endemic settings [38]. Antibody responses to PfMSP3 and PvAMA1 were the least dynamic, influencing the classification of pregnant women into the low immunity cluster suggesting that they are less suitable serological markers of recent exposure to malaria during pregnancy. Conversely, these properties meant that they were the most useful antigens studied in classifying women as controls (i.e. parasite negative) so may have potential for use as part of a broader panel of antigens. Other biomarkers of exposure to $P$. falciparum and $P$. vivax infection have been identified in non-pregnant individuals and would be valuable to investigate in future studies $[39,40]$. This is the first study to incorporate and combine pregnancy and nonpregnancy specific antibodies, so our findings provide a novel avenue for improved sero-surveillance studies to be validated in a range of transmission settings. A further consideration in the application of sero-surveillance is the longevity of antibodies. In this study population, we previously estimated that PfVAR2CSA antibodies may have a very long half-life ( $30-50$ years), whereas PfAMA1 antibodies decay more rapidly (half-life $2-3$ years) [18]. Inclusion of long lived antibody responses, such as PfVAR2CSA, should be interpreted with caution as markers of malaria exposure.

Several methods have been developed to cluster individuals based on longitudinal data, but the majority can only cluster a single longitudinal trajectory from each individual [41-45] or assume that multiple longitudinal trajectories from a single individual are independent [46] and cannot allow samples taken at irregularly (or unevenly) spaced time points [47]. The multivariate mixture linear mixed model approach was selected because it can handle all the statistical complexities/issues posed by the antibody data in this study, i.e., it allows classification of irregularly sampled multivariate longitudinal antibody response data, it is not constrained to assume independence between the longitudinal antibody response profiles from an individual and covariates can be included on the mixture model parameters [31].

This study has several limitations to consider. First, the availability of only one antibody marker for $P$. vivax, PvAMA1, limits what can be understood about the immune response of pregnant women exposed to vivax malaria. The antibody responses to PvAMA1 remained relatively low compared to other antibodies (except for PfMSP3, the only synthetic peptide antigen included in the study which may explain the lower immunogenicity of this $P$. falciparum antigen). Parasite density is believed to have a proportional relationship with antibody maintenance and boosting [48-50]. That these densities are lower in vivax infections compared to falciparum infections [51] could possibly explain the maintenance of PvAMA1 antibody responses at low levels. Administration of chloroquine, preventing vivax episodes, could be a significant confounding factor in disrupting antibody responses to PvAMA1 [24]. The association of chloroquine prophylaxis with $P v A M A 1$ responses was observed though to be in the counterintuitive direction, however, there were a greater proportion of women in the chloroquine prophylaxis arm with a history of $P$. vivax infection 
which increased marginally the PvAMA1 response. This study also only assessed six antibody responses and one domain of VARCSA, therefore, there may be other antigens that are better candidates for sero-surveillance.

Another limitation of this study was that the detection of exposure to malaria was based on microscopy, thereby, failing to detect low density sub-microscopic infections [52]. However, women in the study were tested for malaria infections by microscopy at multiple timepoints during pregnancy, potentially reducing the likelihood of missing low density infections. Pregnant women who regularly access antenatal care comprise an attractive sentinel surveillance population to estimate malaria transmission. Indeed, malaria incidence data from pregnant women spanning 10 years at the very same antenatal clinics was shown to correlate with presentation of clinical malaria in children under 5 years of age [53]. However, further studies are required to determine the utility of this sentinel surveillance population to monitor malaria transmission and if this surveillance method is more sensitive than multiple blood smears to detect a low level parasitaemia. Additionally the generalizability of these antigen combinations to accurately detect exposure to submicroscopic infections needs to be determined in future studies given the high rates of submicroscopic infections in malaria endemic regions [54].

\section{Conclusions}

This study demonstrates that the combination of antiPfAMA1 and anti-PfVAR2CSA antibodies could be used as a potential biomarker of exposure to malaria during pregnancy. However, only $63 \%$ of the malaria infections during pregnancy were detected, therefore further investigation of other antibody markers is warranted. This combined antibody diagnostic tool may facilitate the detection of microscopic infections as an alternative to standard diagnostic methods, particularly in low transmission settings. This proposed malaria sero-surveillance tool may also enhance malaria control and progress efforts to eliminate malaria.

\begin{abstract}
Abbreviations
AMA1: Apical membrane antigen 1; ANCs: Antenatal clinics; CQ: Chloroquine; Crl: Credible interval; Cl: Confidence interval; CSA: Chondroitin sulphate A; EBA: Erythrocyte binding antigen; IEs: Infected erythrocytes; IQR: Inter-quartile range; LOESS: Locally estimated scatterplot smoothing; MCMC: Markov chain Monte Carlo; MSP: Merozoite surface protein; SMRU: Shoklo Malaria Research Unit; VAR2CSA: Variant surface antigen 2-CSA.
\end{abstract}

\section{Supplementary Information}

The online version contains supplementary material available at https://doi. org/10.1186/s12936-022-04111-y.
Additional file 1: Supplementary methods. Table S1. Number and species of infections. Table S2. Penalized expected deviance (PED) values of the models with mixture distributions ranging from $K=1,2,3$, 4 clusters. Table S3. Characteristics of the pregnant women by low and high immune antibody response profile clusters. Table $\mathbf{S 4}$. Assessment of the importance of combining PVAMA1 and PFMSP3, respectively with other P. falciparum antibodies. Table $\mathbf{S 5}$. The importance of including at least PFMSP3 or PVAMA1 with other Plasmodium falciparum antibodies investigated. Table S6. Classification of pregnant women based on the joint antibody responses of PFAMA1 and PFVAR2CSA. Table S7. Classification of pregnant women based on the joint antibody responses of PfAMA1 and PfEBA175, and PfEBA175 and PFVAR2CSA, respectively. Table S8. Classification of cases by the type of infection, based on antibody responses to PFAMA1 and PFVAR2CSA. Figure S1. Longitudinal antibody levels against six antigens for all women free from malaria (controls) by the serostatus to schizont extract. Figure $\mathbf{S 2}$. Longitudinal antibody levels against six antigens for all women by gravidity (primigravidae versus multigravidae). Figure S3. Posterior cumulative distribution functions of the observed data deviances for models with $K=1,2,3,4$ clusters.

\section{Acknowledgements}

We would like to thank the staff of the Shoklo Malaria Research Unit for supporting data collection activities and technical assistance provided in all stages of the study, and Nadia Cross for assistance with generating antibody data in the original study. We are also grateful to all the mothers for their participation.

\section{Authors' contributions}

TTD analysed the data, interpreted the findings and wrote the initial draft of the manuscript; JAS designed the study, supervised the entire study and reviewed the manuscript; FJIF, JGB and KO contributed to study design, data collection, generated antibody data, provided immunological advice and reviewed the manuscript; SD coordinated interpretation of the findings and reviewed the manuscript; SZ contributed to the statistical analyses and reviewed the manuscript, DJP contributed to $R$ coding and reviewed the manuscript, and FN and RM contributed to study design, data collection and reviewed the manuscript. All authors read and approved the final manuscript.

\section{Funding}

A.D.V. Tharkeshi T. Dharmaratne is funded by the University of Melbourne (Melbourne Research Scholarship) for education. The funder had no role in the design of the study, collection, analysis and interpretation of data or writing the manuscript. This work is supported in part by the Australian Centre for Research Excellence in Malaria Elimination, funded by the NHMRC (1134989). JAS is funded by an Australian National Health and Medical Research Council of Australia (NHMRC) Investigator Grant (1196068), FJIF by a NHMRC Career Development Fellowship (1166753), and JGB by a NHMRC Investigator Grant (1173046). The Shoklo Malaria Research Unit is part of the Wellcome Trust Mahidol University Oxford Tropical Medicine Research Unit, which is funded by the Wellcome Trust 220211. For the purpose of Open Access, the author has applied a CC BY public copyright licence to any Author Accepted Manuscript version arising from this submission.

\section{Availability of data and materials}

The datasets used and/or analysed during the current study are available from the corresponding author and study investigators on reasonable request.

\section{Declarations}

\section{Ethics approval and consent to participate}

The study was approved by the Ethics Committee of the Faculty of Tropical Medicine of Mahidol University, the London School of Hygiene and Tropical Medicine, and the Walter and Eliza Hall Institute of Medical Research.

\section{Consent for publication \\ Not applicable.}

\section{Competing interests}

The authors declare that they have no competing interests. 


\begin{abstract}
Author details
${ }^{1}$ Centre for Epidemiology and Biostatistics, Melbourne School of Population and Global Health, The University of Melbourne, Melbourne, Australia. ${ }^{2}$ Burnet Institute, Melbourne, Australia. ${ }^{3}$ Doherty Institute, Melbourne, Australia. ${ }^{4}$ Central Clinical School and Department of Microbiology, Monash University, Melbourne, Australia. ${ }^{5}$ Department of Infectious Diseases, University of Melbourne, Melbourne, Australia. ${ }^{6}$ Shoklo Malaria Research Unit, Mahidol-Oxford Tropical Medicine Research Unit, Faculty of Tropical Medicine, Mahidol University, Mae Sot, Tak, Thailand. ${ }^{7}$ Centre for Tropical Medicine and Global Health, Nuffield Department of Medicine, University of Oxford, Oxford, UK. ${ }^{8}$ Department of Epidemiology and Preventive Medicine, Monash University, Melbourne, Australia.
\end{abstract}

Received: 7 September 2021 Accepted: 28 February 2022 Published online: 05 March 2022

\section{References}

1. WHO. World Malaria Report 2020. Geneva: World Health Organization; 2020

2. Kourtis AP, Read JS, Jamieson DJ. Pregnancy and infection. N Engl J Med. 2014;370:2211-8.

3. Rogerson SJ, Desai M, Mayor A, Sicuri E, Taylor SM, van Eijk AM. Burden, pathology, and costs of malaria in pregnancy: new developments for an old problem. Lancet Infect Dis. 2018;18:e107-18.

4. Dellicour S, Tatem AJ, Guerra CA, Snow RW, ter Kuile FO. Quantifying the number of pregnancies at risk of malaria in 2007: a demographic study. PLoS Med. 2010;7:e1000221.

5. Bauserman M, Conroy AL, North K, Patterson J, Bose C, Meshnick S. An overview of malaria in pregnancy. Semin Perinatol. 2019;43:282-90.

6. Harrington WE, Moore KA, Min AM, Gilder ME, Tun NW, Paw MK, et al. Falciparum but not vivax malaria increases the risk of hypertensive disorders of pregnancy in women followed prospectively from the first trimester. BMC Med. 2021:19:98

7. Mayor A, Menéndez C, Walker PGT. Targeting pregnant women for malaria surveillance. Trends Parasitol. 2019;35:677-86.

8. Ataide R, Mayor A, Rogerson SJ. Malaria, primigravidae, and antibodies: knowledge gained and future perspectives. Trends Parasitol. 2014;30:85-94

9. Elliott SR, Fowkes FJ, Richards JS, Reiling L, Drew DR, Beeson JG. Research priorities for the development and implementation of serological tools for malaria surveillance. F1000 Prime Rep. 2014;6:100.

10. The malERA Consultative Group of Diagnoses and Diagnostics. A research agenda for malaria eradication: diagnoses and diagnostics. PLoS Med. 2011;8:e1000396.

11. Hsiang MS, Hwang J, Kunene S, Drakeley C, Kandula D, Novotny J, et al. Surveillance for malaria elimination in Swaziland: a national cross-sectional study using pooled PCR and serology. PLoS ONE. 2012;7:e29550.

12. Khunrae P, Dahlbäck M, Nielsen MA, Andersen G, Ditlev SB, Resende M, et al. Full-length recombinant Plasmodium falciparum VAR2CSA binds specifically to CSPG and induces potent parasite adhesion-blocking antibodies. J Mol Biol. 2010;397:826-34.

13. Doritchamou J, Sossou-tchatcha S, Cottrell G, Moussiliou A, Hounton Houngbeme C, Massougbodji A, et al. Dynamics in the cytoadherence phenotypes of Plasmodium falciparum infected erythrocytes isolated during pregnancy. PLOS ONE. 2014;9:e98577.

14. Staalsoe T, Shulman CE, Bulmer JN, Kawuondo K, Marsh K, Hviid L. Variant surface antigen-specific lgG and protection against clinical consequences of pregnancy-associated Plasmodium falciparum malaria. Lancet. 2004;363:283-9.

15. O'Neil-Dunne I, Achur RN, Agbor-Enoh ST, Valiyaveettil M, Naik RS, Ockenhouse CF, et al. Gravidity-dependent production of antibodies that inhibit binding of Plasmodium falciparum-infected erythrocytes to placental chondroitin sulfate proteoglycan during pregnancy. Infect Immun. 2001;69:7487-92

16. Fried M, Nosten F, Brockman A, Brabin BJ, Duffy PE. Maternal antibodies block malaria. Nature. 1998;395:851-2.

17. Cutts JC, Agius PA, Zaw L, Powell R, Moore K, Draper B, et al. Pregnancyspecific malarial immunity and risk of malaria in pregnancy and adverse birth outcomes: a systematic review. BMC Med. 2020;18:14.
18. Fowkes FJ, McGready R, Cross NJ, Hommel M, Simpson JA, Elliott SR, et al. New insights into acquisition, boosting, and longevity of immunity to malaria in pregnant women. J Infect Dis. 2012;206:1612-21.

19. Lloyd YM, Fang R, Bobbili N, Vanda K, Ngati E, Sanchez-Quintero MJ, et al. Association of antibodies to VAR2CSA and merozoite antigens with pregnancy outcomes in women living in Yaoundé. Cameroon Infect Immun. 2018:86:e00166-e218.

20. Luxemburger C, Ricci F, Nosten F, Raimond D, Bathet S, White NJ. The epidemiology of severe malaria in an area of low transmission in Thailand. Trans R Soc Trop Med Hyg. 1997;91:256-62.

21. Parker DM, Carrara VI, Pukrittayakamee S, McGready R, Nosten FH. Malaria ecology along the Thailand-Myanmar border. Malar J. 2015;14:388.

22. Nosten F, McGready R, Simpson JA, Thwai KL, Balkan S, Cho T, et al. Effects of Plasmodium vivax malaria in pregnancy. Lancet. 1999;354:546-9.

23. Nosten F, ter Kuile F, Maelankirri L, Decludt B, White NJ. Malaria during pregnancy in an area of unstable endemicity. Trans R Soc Trop Med Hyg. 1991;85:424-9

24. Villegas L, McGready R, Htway M, Paw MK, Pimanpanarak M, Arunjerdja $\mathrm{R}$, et al. Chloroquine prophylaxis against vivax malaria in pregnancy: a randomized, double-blind, placebo-controlled trial. Trop Med Int Health. 2007;12:209-18.

25. Mugyenyi CK, Elliott SR, Yap XZ, Feng G, Boeuf P, Fegan G, et al. Declining malaria transmission differentially impacts the maintenance of humoral immunity to Plasmodium falciparum in children. J Infect Dis. 2017;216:887-98.

26. Yildiz Zeyrek F, Palacpac N, Yuksel F, Yagi M, Honjo K, Fujita Y, et al. Serologic markers in relation to parasite exposure history help to estimate transmission dynamics of Plasmodium vivax. PLOS ONE. 2011;6:e28126.

27. Stanisic DI, Richards JS, McCallum FJ, Michon P, King CL, Schoepflin S, et al. Immunoglobulin G subclass-specific responses against Plasmodium falciparum merozoite antigens are associated with control of parasitemia and protection from symptomatic illness. Infect Immun. 2009;77:1165-74.

28. Richards JS, Stanisic DI, Fowkes FJ, Tavul L, Dabod E, Thompson JK, et al. Association between naturally acquired antibodies to erythrocyte-binding antigens of Plasmodium falciparum and protection from malaria and high-density parasitemia. Clin Infect Dis. 2010;51:e50-60.

29. Roussilhon C, Oeuvray C, Müller-Graf C, Tall A, Rogier C, Trape JF, et al. Long-term clinical protection from falciparum malaria is strongly associated with lgG3 antibodies to merozoite surface protein 3. PLoS Med. 2007:4:e320.

30. Cutts JC, Powell R, Agius PA, Beeson JG, Simpson JA, Fowkes FJ. Immunological markers of Plasmodium vivax exposure and immunity: a systematic review and meta-analysis. BMC Med. 2014;12:150.

31. Komárek A, Komárková L. Clustering for multivariate continuous and discrete longitudinal data. Ann Appl Stat. 2013;7:177-200.

32. Komárek A, Komárková L. Capabilities of R Package mixAK for clustering based on multivariate continuous and discrete longitudinal data. J Stat Softw. 2014;59:1-38.

33. Asparouhov T, Muthén B. Variable-specific entropy contribution. 2014 http://www.statmodel.com/download/UnivariateEntropy.pdf.

34. Kitojo C, Gutman JR, Chacky F, Kigadye E, Mkude S, Mandike R, et al. Estimating malaria burden among pregnant women using data from antenatal care centres in Tanzania: a population-based study. Lancet Glob Health. 2019;7:e1695-705.

35. Fonseca AM, González R, Bardají A, Jairoce $C$, Rupérez M, Jiménez A, et al. VAR2CSA serology to detect Plasmodium falciparum transmission pat terns in pregnancy. Emerg Infect Dis. 2019;25:1851-60.

36. Stanisic DI, Fowkes FJ, Koinari M, Javati S, Lin E, Kiniboro B, et al. Acquisition of antibodies against Plasmodium falciparum merozoites and malaria immunity in young children and the influence of age, force of infection, and magnitude of response. Infect Immun. 2015;83:646-60.

37. Ibison F, Olotu A, Muema DM, Mwacharo J, Ohuma E, Kimani D, et al. Lack of avidity maturation of merozoite antigen-specific antibodies with increasing exposure to Plasmodium falciparum amongst children and adults exposed to endemic malaria in Kenya. PLoS ONE. 2012;7:e52939.

38. Rijken MJ, McGready R, Boel ME, Poespoprodjo R, Singh N, Syafruddin D, et al. Malaria in pregnancy in the Asia-Pacific region. Lancet ID. 2012:12:75-88.

39. Longley RJ, White MT, Takashima E, Brewster J, Morita M, Harbers M, et al. Development and validation of serological markers for detecting recent Plasmodium vivax infection. Nat Med. 2020;26:741-9. 
40. Helb DA, Tetteh KK, Felgner PL, Skinner J, Hubbard A, Arinaitwe E, et al. Novel serologic biomarkers provide accurate estimates of recent Plasmodium falciparum exposure for individuals and communities. Proc Natl Acad Sci USA. 2015;112:E4438-47.

41. Tarpey T, Kinateder K. Clustering functional data. J Classification. 2003;20:93-114.

42. Abraham C, Cornillon PA, Matzner-Løber E, Molinari N. Unsupervised curve clustering using B-splines. Scand J Statist. 2003;30:581-95.

43. James GM, Sugar CA. Clustering for sparsely sampled functional data. J Am Stat Assoc. 2003;98:397-408.

44. Genolini C, Falissard B. KmL: a package to cluster longitudinal data. Comput Methods Progr Biomed. 2011;104:e112-21.

45. Sylvestre MP, McCusker J, Cole M, Regeasse A, Belzile E, Abrahamowicz M. Classification of patterns of delirium severity scores over time in an elderly population. Int Psychogeriatr. 2006;18:667-80.

46. Grün B, Leisch F. FlexMix Version 2: finite mixtures with concomitant variables and varying and constant parameters. J Stat Softw. 2008;28:1-35.

47. McNicholas PD, Murphy TB. Model-based clustering of longitudinal data. Canad J Stat. 2010;38:153-68.

48. McCallum FJ, Persson KE, Fowkes FJ, Reiling L, Mugyenyi CK, Richards $J$ S, et al. Differing rates of antibody acquisition to merozoite antigens in malaria: implications for immunity and surveillance. J Leukoc Biol. 2017;101:913-25.

49. Kerkhof K, Sluydts V, Willen L, Kim S, Canier L, Heng S, et al. Serological markers to measure recent changes in malaria at population level in Cambodia. Malar J. 2016;15:529.

50. van den Hoogen LL, Walk J, Oulton T, Reuling IJ, Reiling L, Beeson JG, et al. Antibody responses to antigenic targets of recent exposure are associated with low-density parasitemia in controlled human Plasmodium falciparum infections. Front Microbiol. 2018;9:3300.

51. Karunajeewa HA, Mueller I, Senn M, Lin E, Law I, Gomorrai PS, et al. A trial of combination antimalarial therapies in children from Papua New Guinea. N Engl J Med. 2008;359:2545-57.

52. Kattenberg JH, Ochodo EA, Boer KR, Schallig HD, Mens PF, Leeflang MM. Systematic review and meta-analysis: rapid diagnostic tests versus placental histology, microscopy and PCR for malaria in pregnant women. Malar J. 2011;10:321.

53. Nkhoma SC, Nair S, Al-Saai S, Ashley E, McGready R, Phyo AP, et al. Population genetic correlates of declining transmission in a human pathogen. Mol Ecol. 2013;22:273-85.

54. Okell LC, Ghani AC, Lyons E, Drakeley CJ. Submicroscopic infection in Plasmodium falciparum-endemic populations: a systematic review and meta-analysis. J Infect Dis. 2009;200:1509-17.

\section{Publisher's Note}

Springer Nature remains neutral with regard to jurisdictional claims in published maps and institutional affiliations.

Ready to submit your research? Choose BMC and benefit from:

- fast, convenient online submission

- thorough peer review by experienced researchers in your field

- rapid publication on acceptance

- support for research data, including large and complex data types

- gold Open Access which fosters wider collaboration and increased citations

- maximum visibility for your research: over $100 \mathrm{M}$ website views per year

At BMC, research is always in progress.

Learn more biomedcentral.com/submissions 